\title{
Topical Lidocaine and Epidural Bupivacaine/ Hydromorphone in the Treatment of Complex Regional Pain Syndrome Type 2
}

\section{TO THE EDITOR:}

We report a case of Complex Regional Pain Syndrome (CRPS) type 2 that was successfully treated with a lumbar epidural bupivacaine/hydromorphone infusion and lidocaine $5 \%$ patch to the affected foot. Few reports discuss these options in the treatment of CRPS type 2 and further investigation may be warranted.

A 41-year-old otherwise healthy female noted the insidious onset of left leg radicular pains that led to the diagnosis of a sacral chondrosarcoma that was resected. Her preoperative course was complicated by a venous thrombosis in the left leg that was treated with anticoagulation and an IVC filter. During the resection, the left $\mathrm{S} 1$ nerve root was sacrificed. The patient noted a progressive burning dysesthesia in the left foot postoperatively that required readmission 6 weeks later. Pain rating was 10 out of 10 on the visual analogue scale at readmission. General examination and the surgical site were unremarkable. Even though sensory and motor findings were consistent with an S1 root lesion, the patient experienced pain in the entire foot well beyond S1 dermatome. The foot was erythematous, warm, slightly edematous, and demonstrated marked mechanical allodynia throughout. Pulses were strong and symmetric. There was no significant edema, trophic changes, or tenderness over the calf. Pregabilin, tizanidine, benzodiazepines, mirtazapine, and opiates weren't helpful, so a lumbar epidural catheter was placed and an infusion of $0.125 \%$ bupivacaine and $10 \mathrm{mcg} / \mathrm{mL}$ of hydromorphone at $6 \mathrm{~mL} / \mathrm{hr}$ was begun. Pain was improved the next day to 5 out of 10 . A lidocaine $5 \%$ topical patch was then applied and by the following day her pain was just one out of 10. Three days later she had minimal allodynia and decreased erythema and was discharged from the hospital. Follow up in the surgical center has found her to be doing very well with minimal pain. She still uses the lidocaine patch in addition to tizanindine, pregabalin, and oxycodone/acetaminophen as needed. She is off sustained release oxycodone.

Numerous medications have been tried to treat CRPS with varying success (1). One open label study of lidocaine $5 \%$ topical patches demonstrated an effect on patients with CRPS (2), but other reports of topical lidocaine's utility in this condition are scarce. Likewise, little information regarding epidural analgesia with local anesthetics or opiates as a treatment of CRPS is to be found, though there does exist reference to epidural injections as a sympatholytic option (3) and to epidural infusion as a preventative measure perioperatively (4). Our patient had such a significant response to these 2 modalities that it seemed worth reporting to garner attention to such interventions.

Regarding the diagnostic impression that this was in fact CRPS, a few points should be made. Her case is confounded by venous thrombosis in the ipsilateral leg, infectious/inflammatory mimics weren't ruled out, and neither nuclear isotope imaging nor MRI was performed on the affected foot. Furthermore, she was on other medications that may have contributed to pain control, and one could argue that the case represents a traumatic $\mathrm{S1}$ radiculopathy. We felt that the thrombosis wasn't the source of her pain because it had been present preoperatively and was being treated prior to the development of her burning dysesthetic pain. Infection and non-infectious inflammatory disorders seem unlikely as she improved in the absence of antibiotic or anti-inflammatory medications. Though we requested a bone scan and MRI with gadolinium, these weren't performed and treatment was more urgent than diagnostic testing that are not necessarily sensitive or specific for CRPS (5). Regarding her other medications, the strongest temporal correlation with her improvement was with the epidural medications and topical lidocaine. Finally, her pain included but also extended beyond the $\mathrm{S} 1$ dermatome, involving the entire foot.

From a pathophysiologic standpoint, this case may provide insight into the mechanisms by which CRPS type 2 develops. A variety of pain related phenomena, both central and peripheral, have been associated with the development of neuropathic pain. A continuum starting with "irritability" of the nociceptive system at one end and almost complete deafferentation on the other end has been demonstrated in the same neu- 
ropathic pain disorder, postherpetic neuralgia $(6,7)$. Several features of peripheral sensitization occur early during PHN, including a reduction in the threshold of pain caused by heat at the site of erythematous skin, a phenomenon that disappears later in the natural history of the disease when abnormal responses in the central nervous system dominate the clinical picture (8). It is probably not a coincidence that the lidocaine $5 \%$ patch, a topical preparation with minimal systemic absorption, is more effective in postherpetic neuralgia than in many other neuropathic pain conditions where the peripheral sensitization doesn't play such a significant role in the pain manifestation (9).

Central sensitization is the most likely physiological phenomenon that underlines the clinical spectrum of pain features seen in CRPS (10). Central sensitization amplifies and facilitates the synaptic transfer from the nociceptor central terminal to dorsal horn neurons. During the late phase of central sensitization, it is sustained beyond the initiating stimulus by transcriptional changes in the molecular machinery of the cell (11). That may explain a relative lack of efficacy of medications targeting peripheral mechanisms of neuropathic pain seen in many patients with established CRPS. However, in CRPS type 2, with its distinct peripheral nerve (or dorsal root, as in the presented case) injury, peripheral sensitization might play a much more important role in the development of this usually recalcitrant pain syndrome, compared to CRPS type 1 when the initial event is often minor or, sometimes, even can't be recalled by the patient. Therefore, the pathogenesis of CRPS type 2 may not be dissimilar to that of postherpetic neuralgia that starts with the peripheral sensitization process, and then proceeds through the acute phase of central sensitization to the full-fledged late phase of central sensitization associated with intractable spreading pain poorly responsive to topical preparations. It has been demonstrated that the early stage of central sensitization, which our case of nascent CRPS type 2 might represent, is actively dependent on nociceptive input into the spinal cord $(12,13)$. Thus, such agents as lidocaine $5 \%$ patch or/ and prolonged regional block with local anesthetic in the epidural space could interrupt the sensory barrage from the periphery which maintains the acute central sensitization and, therefore, might be proven of great value if used in the very early stages of developing CRPS type 2.

The above case suggests that application of local anesthetics to sites along the peripheral nervous system and into the epidural space early in the course of CRPS type 2 may be able to shut down the process by which the disorder establishes itself. That these 2 treatments seemed so effective warrants more investigation and may suggest a strong peripheral component to the initial stages of CRPS type 2 .

Yakov Vorobeychik, MD

Assistant Professor of Anesthesiology and Neurology Milton S. Hershey Medical Center

500 University Drive, HU32

Hershey, PA 17033

e-mail: yvorobeychik@psu.edu

David M. Giampetro, MD

Assistant Professor of Anesthesiology and Neurology Milton S. Hershey Medical Center

500 University Drive, HU32

Hershey, PA 17033

\section{References}

1. Kingery WS. A critical review of controlled clinical trials for peripheral neu- 6 . ropathic pain and complex regional pain syndrome. Pain 1997; 73:123-139.

2. Devers A, Galer BS. Topical lidocaine patch relieves a variety of neuropath- 7 ic pain conditions: an open-label study. Clin J Pain 2000; 16:205-208.

3. Gordon N. Reflex sympathetic dystrophy. Brain \& Development 1996; 18:257- 8 262.

4. Reuben, SS. Preventing the development of complex regional pain syndrome after surgery. Anesthesiology 9 2004; 101:1215-1224

5. Bruehl S, Harden RN, Galer BS, Saltz S, Backonja M, Stanton-Hicks M. Complex regional pain syndrome: are there distinct subtypes and sequential stages of the syndrome? Pain 2002; 95:119-124. Fields HL, Rowbotham M, Baron R. Postherpetic neuralgia: irritable nociceptors and deafferentation. Neurobiol Dis 1998; 5:209-227.

7. Rowbotham M. Mechanisms of neuropathic pain and their implications for the design of clinical trials. Neurology 2005; 65(Suppl 4):S66-S73.

8. Rowbotham M, Fields HL. The relationship of pain, allodynia and thermal sensation in post-herpetic neuralgia. Brain 1996; 119:347-354.

Dworkin R, Backonja M, Rowbotham M, Allen RR, Argoff CR, Bennet GJ, Bushnell MC, Farrar JT, Galer BS, Haythornthwaite JA, Hewitt DJ, Loeser JD, Max MB, Saltarelli M, Schmader KE, Stein C, Thompson D, Turk DC, Wallace MS,
Watkins LR, Weinstein SM. Advances in neuropathic pain. Arch Neurol 2003; 60:1524-1534.

10. Schwartzman R, Vorobeychik Y. Complex regional pain syndrome I/II. In: Evans R (ed): Neurology and Trauma. New York. Oxford University Press 2006; pp. 465-476.

11. Ji RR, Kohno T, Moore KA, Woolf CJ. Central sensitization and LTP: do pain and memory share similar mechanisms? TINS 2003; 26:696-705.

12. Woolf CJ, Salter MW. Neuronal plasticity: increasing the gain in pain. Science 2000; 228:1765-1769.

13. Woolf $\mathrm{CJ}$. Pain: moving from symptom control toward mechanism-specific pharmacologic management. Ann Intern Med 2004; 140:441-451. 